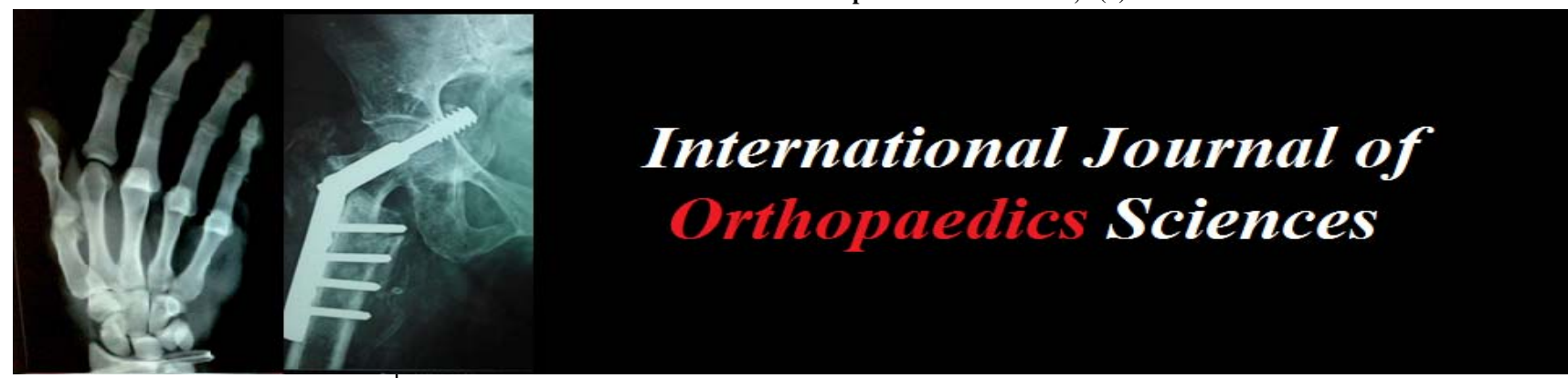

ISSN: $2395-1958$

IJOS 2017; 3(2): 76-79

(C) 2017 IJOS

www.orthopaper.com

Received: 14-02-2017

Accepted: 15-03-2017

Dr. Dayal Chavan

Fellow in Arthroplasty and

Arthroscopy, Hosmat Hospital,

Bangalore, India

Dr. Srinivas Nagendra G

Associate Professor, Dept. of orthopedics, Sapthagiri Institute of Medical sciences, Bangalore

\section{Outcome of total hip replacement after failed bipolar hemiarthroplasty for fracture neck of femur}

\author{
Dr. Dayal Chavan and Dr. Srinivas Nagendra G
}

DOI: http://dx.doi.org/10.22271/ortho.2017.v3.i2b.14

Abstract

Introduction: The most common indication for conversion total hip replacement after previous hemiarthroplasty is pain. Pain can result from loosening of the femoral stem, erosion of the acetabulum, a combination of the two and sepsis. More recently, two additional indications are disassembly and wear of modular hemiarthroplasty components

Methodology: A total 22 patients who had conversion of their failed hemiarthroplasty to a total hip replacement in Hospital were analyzed retrospectively and prospectively. Those patients who had been operated with hemiarthroplasty for diagnosis other than fracture neck of femur were excluded from the study. All patients were evaluated clinically and radiologically with review of serial follow-up radiography if available to identify the possible cause of failure

Results: The mean leg length discrepancy was $11 \mathrm{~mm}$ seen in four patients. Complications occurred in 4 patients (18\%). One patient had early wound infection which cured completely after debridement, suction-irrigation and 6 weeks of intravenous antibiotics. One patient had incomplete sciatic nerve lesion which recovered completely at one year follow-up. Two patients had persistent groin pain. We had no loosening at last follow-up. Also neither instability nor mortality was reported.

Conclusion: Conversion of painful hemiarthroplasty gives good results with regard to the pain relief and functional scores

Keywords: Total HIP replacement, hemiarthroplasty, fracture neck of femur

\section{Introduction}

Hemiarthroplasty has been widely used for the treatment of displaced intracapsular fractures of the femoral neck because of the high incidence of nonunion and avascular necrosis associated with reduction and internal fixation. Inevitably, some hemiarthroplasties fail and require revision. The most common indication for conversion total hip replacement after previous hemiarthroplasty is pain ${ }^{[1]}$. Pain can result from loosening of the femoral stem, erosion of the acetabulum, a combination of the two and sepsis. More recently, two additional indications are disassembly and wear of modular hemiarthroplasty components. One of the most commonly encountered clinical situations is the need for conversion surgery in patients received reconstruction with Austin-Moore type prosthesis for displaced fractures of the femoral neck. Subsidence of the prosthesis is common, but many patients with such subsidence can remain clinically satisfied ${ }^{[2]}$. Erosion of the acetabular cartilage, however, has been shown to be one of the primary mechanisms resulting in significant pain the necessitates conversion surgery to total hip replacement. Whittaker et al. reported a $64 \%$ incidence of loss of acetabular cartilage, with a $24 \%$ incidence of development of protrusion aceabuli at 15 years after hemiarthroplasty surgery. Kofoed and Kofod reported a 37\% incidence of conversion to total hip replacement within the first 2 years after hemiarthroplasty using the Austin-Moore prosthesis in 106 patients with femoral neck features. Dalldrof et al. reported evidence of actebular cartilage degeneration using histology of biopsied specimens during conversion surgery in those patients who had previously undergone hemiarthroplasty surgery. They found a significant difference in the histology of the cartilage when compared to a group of control patients who were undergoing primary hip replacement surgery for femoral neck fractures. Moreover, the degree of cartilage degeneration was significantly related to the during of articulation with the metallic implant ${ }^{[3-5]}$. The fate of the acetabular cartilage that had articulated with a unipolar 
prosthesis was similar to that articulated with a bipolar Prosthesis ${ }^{[6]}$. The incidence of subsidence and radiographic loosening of the stem were similar for those patients who went on to the conversion surgery and for those who remained clinically satisfied the most significant finding was that the presence of acetabular degeneration was 4 time grater in those patients who underwent conversion surgery. Another important finding in that study was that over two thirds of those patients needing conversion surgery were younger and more active.

\section{Methodology}

Total 22 patients who had conversion of their failed hemiarthroplasty to a total hip replacement in Hospital were analyzed retrospectively and prospectively. Those patients who had been operated with hemiarthroplasty for diagnosis other than fracture neck of femur were excluded from the study. All patients were evaluated clinically and radiologically with review of serial follow-up radiographys if available to identify the possible cause of failure. Investigations conducted in clinic included a septic screen (full blood count, ESR, CRP) and pain anteroposterior and lateral X-rays of the symptomatic hip. Treatment strategies prior to conversion were also reviewed. All the patients received perioperative antibiotic prophylaxis.

Radiological examination assessed abnormal position of the prosthesis, inadequate contact with the calar, descent of the prosthesis into the femur, radiolucencies around the prosthesis and protrusion acetabulae (medial migration of the prosthetic head beyond Kohler's line) Change in component position or progression of lucent lines were reported in serial follow-up radiographs according to the zones of Gruen et al for the femur and De Lee and Charneley for the acetabulum. We used the criteria of Olsson for diagnosis of loosening. Distance between lesser trochanter and prosthesis collar was used to measure femoral component subsidence as compared to first postoperative X-ray. Harris hip score was used for functional evaluation both pre-and postoperatively.

The main reasons for conversion arthroplasty were acetabular erosion in 5 patients $(22.7 \%)$. Stem loosening in 8 patients $(36.3 \%)$, both loosening of stem and acetabular in 2 patients (9\%), 4 patients $(18.1 \%)$ with femoral shaft fracture, $2(9 \%)$ patients with deep infection and 1 patient (4.5\%) with protrusion acetabula.

The main time from primary operation to appearance of symptoms in this group of patients was 19 months (range 2-96 months), 11 months for femoral

Loosening (range 5-46 months) and 28 months for acetabular (9-96 months).

The mean time interval from appearance of symptoms to revision surgery for this group of patients was 54 months (4.5years)

A standard posterior approach to the hip was used in all patients. At operation the condition of the particular cartilage $\mathrm{n}$ the acetabulum, the stability of the prosthesis in the proximal femur and any other associated pathological conditions found were documented by the surgeon.

Intraoperatively, stability was examined by observing for dislocation while the hip is being put through the full range of motion possible and by observing for displacement while applying traction to the limb. If the stability was questionable, we attempted to suture the posterior soft tissues more tightly posterior soft tissue repair was performed in all patients using 2-0 Ethibond.

Patients revised for infection had a two stage revision, A transfemoral approach was used in 5 patients: 2 patients with acetabular erosion, 2 with a fractured stem and one with infection. Acetabular grafting with autogenous morsellised ipsilateral iliac bone graft was used in 3 partients, cementless in 19 patients. Low-molecular-weight heparin (Enoxaparin subcutaneously $4000 \mathrm{U}$ once daily) was used for deep venous thrombosis prophyulaxis IV ceftriaxone + Sulbactum was given after tissue culture was taken intra-operatively, and was continued for 2-3 days depending on the culture results.

Static quadriceps exercises and knee and ankle mobilization were started on the day of surgery. Fifteen patients were allowed to walk with tolerable weight bearing with walker or crutches on the day after surgery. 4 Patients with periprosthetic fractures were kept on non-weight bearing crutch walking until the radiological evidence of fracture union, whereas 2 patient with acetabular reconstruction using autogenous morsellised ipsilateral iliac bone grafts were allowed to walk partial weight bearing for first 3 weeks, Results were evaluated using Harris Hip Score the Preoperative Harris Hip Score were obtained from hospital records. Postoperative scores were obtained at each follow-up visit and telephonic interview. All the patients were available for follow-up.

\section{Results}

Of the 22 patients revised, there were 14 women $(63.6 \%)$ and 8 men $(36.4 \%)$ with a mean age of 61 years (range 32 to 79 years) at the time of conversion arthroplasty. The mean period of follow up was 41 months (range 18 to 86months)

Pain with either antalgic gait [groin pain (5), thigh pain (8) or both (2)] or leg length

Discrepancy (5) was the chief presenting complaint. Other presenting complaints are shown in (Table1.)

Table 1: Patients' Complaints

\begin{tabular}{|c|c|c|}
\hline Complaints & $\mathbf{N = 2 2}$ & $\mathbf{\%}$ \\
\hline Pain & 6 & 27.2 \\
\hline Pain+Antalgic gait & 9 & 40.9 \\
\hline Pain +LLD & 4 & 18.2 \\
\hline Pain+antalgic gait+LLD & 3 & 13.6 \\
\hline
\end{tabular}

$\mathrm{LLd}=$ leg length discrepancy

The main time interval from appearance of symptoms to revision surgery for this group of patients was 54 months (4.5years). Seven Austin Moore and 15 Bipolar Prostheses had been implanted for fracture neck of femur in cases that was revised in our series. All the bipolar prostheses that were revised were of the monoblock type. No patient who had undergone Modular Bipolar prosthesis for fracture neck of femur had come for revision. Pain was the leading sign in all patients. The mean follow up period after conversion total hip prosthesis was 41 months (3.5years)

The overall Harris score improved from a mean of 38.4 preoperatively to a mean of 87.7 at one year follow up and to 84.7 points at the last follow-up. Pain scores showed marked improvement: We had 19 patients with no hip pain and 3 patients with slight pain. Also, at the last follow up we had 19 patients walking unaided and 3 patients needed minimal support of cane. Range of Motion improved significantly and was essentially normal in all.

From the radiological examination(Table II) it was determined that the femoral components $(45 \%)$ had more than $2 \mathrm{~mm}$ radiolucent lines in Gruen Zones I, IV and VII Assessment of the acetabulum revealed protrusion in one case and some degree of cartilage erosion in the five, which was also detected during surgery. 
Table 2: Plain X-ray (AP/Lat) results

\begin{tabular}{|c|c|c|}
\hline & $\mathbf{n}$ & $\mathbf{( \%}$ \\
\hline $\begin{array}{c}\text { Femoral loosening (transulucent lines)_50\% around } \\
\text { prosthesis }\end{array}$ & 10 & 45 \\
\hline No calcar contact & 2 & 9 \\
\hline Acetabular erosion & 5 & 23 \\
\hline Protrusion & 1 & 5 \\
\hline Periprosthetic Fracture & 4 & 18 \\
\hline
\end{tabular}

In the last follow-up controls of conversion total hip prosthesis there were no radiolucent lines in either acetabular or femoral components. Radiological evaluation showed good bony in growth and stability of all the femoral components. None of the acetabular component showed migration, loosening, were, or osteolysis at last follow-up.

Operative findings at the time of conversion showed loosening of the prosthesis and acetabular cartilage degeneration as the main pathological processes leading to failure of the hemiarthroplasty (Table III)

Table 3: Operative findings at conversion

\begin{tabular}{|c|c|c|}
\hline & $\mathbf{n}$ & $\mathbf{( \%}$ \\
\hline Femoral loosening & 9 & 41 \\
\hline Acetabular cartilage degeneration & 6 & 27 \\
\hline Femoral loosening and acetabular cartilage degeneration & 3 & 14 \\
\hline Periprosthetic fracture & 4 & 18 \\
\hline
\end{tabular}

Bone defects were detected during the operation in the femur in 2 patients $(9 \%)$, in the acetabulum in one patient $(4.5 \%)$ and cancellous allografts and calcar replacing long stem prosthesis were used for coverage of these defects.

Harris hip scores were affected by the indication for conversion arthroplasty (Table IV)

Table 4: Harris Hip Score in relation to indication

\begin{tabular}{|c|c|c|}
\hline Indications & Preoperative & $\begin{array}{c}\text { Last follow- } \\
\text { up }\end{array}$ \\
\hline Acetabular erosion $(\mathrm{N}=5)$ & 49.6 & 88.1 \\
\hline Fomoral loosening $(\mathrm{N}=8)$ & 41.2 & 85.5 \\
\hline $\begin{array}{c}\text { Acetabular erosion + loosening } \\
(\mathrm{N}=2)\end{array}$ & 26.3 & 84.8 \\
\hline Fomoral fracture $(\mathrm{N}=4)$ & 23.3 & 89.0 \\
\hline Infection $(\mathrm{N}=2)$ & 44.0 & 70.7 \\
\hline Protrusion acetabula(N=1) & 37.6 & 81.0 \\
\hline
\end{tabular}

At last follow-up we had $13.6 \%$ excellent results and $72 \%$ good results, $9 \%$ fair results and $4.5 \%$ poor results at the end of follow-up (Table V)

Table 5: Functional result after conversion arthroplasty

\begin{tabular}{|c|c|c|}
\hline Functional result & Last follow-up No & Last follow-up\% \\
\hline Excellent & 3 & 13.6 \\
\hline Good & 16 & 72.7 \\
\hline Fair & 2 & 9.0 \\
\hline Poor & 1 & 4.5 \\
\hline
\end{tabular}

The mean leg length discrepancy was $11 \mathrm{~mm}$ seen in four patients. (range from $10 \mathrm{~mm}$ to $12 \mathrm{~mm}$ ). Complications occurred in 4 patients $(18 \%)$. One patient had early wound infection which cured completely after debridement, suctionirrigation and 6 weeks of intravenous antibiotics. One patient had incomplete sciatic nerve lesion which recovered completely at one year follow-up. Two patients had persistent groin pain. (Table VI). We had no loosening at last follow-up. Also neither instability nor mortality was reported.
Table 6: Complications in relation to the indications for conversion arthroplasty

\begin{tabular}{|c|c|c|c|c|}
\hline Indication & $\begin{array}{c}\text { Wound } \\
\text { infection }\end{array}$ & $\begin{array}{c}\text { Groin } \\
\text { pain }\end{array}$ & $\begin{array}{c}\text { Neural } \\
\text { deficit }\end{array}$ & Total \\
\hline Acetabular erosion & 0 & 1 & 0 & 1 \\
\hline Fomoral loosening & 0 & 0 & 0 & 0 \\
\hline Acetabular erosion & 0 & 0 & 0 & 0 \\
\hline + loosening & 0 & 0 & 1 & 1 \\
\hline $\begin{array}{c}\text { Fomoral fracture } \\
\text { Infection }\end{array}$ & 1 & 0 & 0 & 1 \\
\hline Protrusion acetabula & 0 & 1 & 0 & 1 \\
\hline Total & 1 & 2 & 1 & 4 \\
\hline
\end{tabular}

\section{Discussion}

Hemiarthroplasty (Unipolar and bipolar) of the hip is a commonly performed procedure for the treatment of displaced intra-articular fractures of the neck of the femur in the elderly. The goal of treatment of displaced fractures of the neck of the femur is to return the patients to their pre-injury mobility status as early as possible and to minimize the risk of further operation. Austin Moore and Thompson prostheses have fulfilled these criteria for decades but have associated with a poor quality of life in the long term with a very high incidence of groin and thigh pain in physically active elderly patients, largely a consequence of acetabular cartilage degeneration and stem loosening respectively ${ }^{[7]}$. In our study group of failed hemiarthroplasty $41 \%$ patients complained of groin pain, $25 \%$ thigh pain and $14 \%$ had both. Bipolar arthroplasty was introduced to improve the long-term outcome of hemiarthroplasty as a result of less wear of the metal cartilage interface by providing another interface (metalpolyethlene) inside the bipolar head. However, recent studies comparing bipolar to unipolar hemiarthroplasty show little difference between the two with regard to morbidity, mortality, or functional outcome. Pain following hemiarthroplasty is usually due to one of two pathological processes: particular cartilage degeneration in the acetabulum or loosening of the prosthesis. These pathological processes are exacerbated by many factors including incongruence between the femoral head and the acetabulum, excessive neck length, impaction at the time of injury, cementation of the prosthesis, physiologically young active patients and shear forces between the prosthesis and the cartilage ${ }^{[8]}$. In view of these observations current evidence is emerging that favors THA over hemiarthroplasty for treatment of displayed fractures of the neck of femur in patients who are elderly but have an active physical life. The treatment of symptomatic hemiarthroplasty involves removal of the prosthesis and conversion to a total hip replacement and Cossey and Goodwin noted that conversion to THA would give satisfactory results. Other investigators, however, have reported that conversion of hemiarthroplasty to THA is associated with high complication and loosening rates as against primary total hip arthroplasty. Groin pain, which has been cited as the most common reason for conversion, does not seem to be relieved completely in every patient after conversion to THA. Sharkey et al., while reporting the results of conversion of hemiarthroplasty to THA in 45 patients observed that $20 \%$ of the patients continued to have groin or buttock pain after THA and they could not identify a factor that would predict an unsuccessful result. In our study group 15 out of $18(83 \%)$ patients who had isolated only partial improvement ${ }^{[9]}$. Thigh pain, however, was relieved in all patients. Six patients who had both groin and thigh pain were relieved of both postoperatively. Sharkey et al., suggested that 
patients should be warned of this contingency before the surgery, that they could experience some groin pain postoperatively. One of the earliest studies on conversion arthroplasty, by Amstutz and Smith, Noted very high incidence of intra as well as postoperative complications. They reported results of 41 patients with conversion arthroplasty. They had five intraoperative proximal femoral fractures, two perforations of the medial cortex with stem protrusion, two cases with instability, two cases with infection, there patients with deep venous thrombosis and six patients $(14.6 \%)$ with progressive loosening. There patients had required revision by the end of follow-up (mean of 36 months). Sierra and Cabanela in a larger series of 132 hemiarthroplasties that were converted to THA reported a $10 \%$ rate of loosening after a mean follow-up of 7.1 years and major complications in $45 \%$, including 12 endoprostheses to THAs after femoral neck fractures is fraught with high complication and loosening rates and careful selection of patients for each type of arthroplasty (hemiarthroplasty versus THA) may help ameliorate the outcome of arthroplasty for this group of patients. We also observed a high rate of intraoperative complications with iatrogenic fracture of the femur in two, femoral performation in two, partial trochanteric avulsion in two and fracture of the acetabular floor in three hips although none of these complications resulted in poor long-term outcome ${ }^{[10]}$. The rate loosening in our series was $2.3 \%$ (one out of 44) after a mean follow-up of 6.4 years. Hammad and AbdelAal reported no loosening in their series of conversion arthroplasty in 47 patients after an average follow-up of 44 months. The reason for lower loosening rate in their series as against earlier studies, as stated by them may have been as a result of better cementing technique and stem design. In addition, failure on the femoral side may be due extensive resorption of the endosteal bone while the stem of the hemiarthroplasty was loose, or due to damage of the endosteal bone during revision. Furthermore, toggling of the stem may produce a thick fibrous membrane that is adherent and might not be completely removed at revision, with its remnants compromising the subsequent cemented fixation. Also, it had been suggested that fragments of such a fibrous membrane are metabolically very active, producing Prostaglandin e2, collagenase and interleukin $1 \mathrm{~b}$, all of which may contribute to resorption of adjacent bone. Our series differs from these studies in one respect i.e. all acetabular components and the majority $(86.5 \%)$ of femoral components used in our series were uncemented and this was probably the reason for lower loosening rates. The incidence of dislocation after conversion arthroplasty has been reported as varying from 0 to $50 \%$ in different series. We had one dislocation in the early postoperative period and we believe that occurrence of postoperative instability is technique-related. Conversion of painful hemiarthroplasty gives good results with regard to the pain relief and functional scores. Our functional results were very encouraging with an average HHS of 86 at final follow-up and $22(50 \%)$ patients were community ambulators without support while $17(38 \%)$ patients could walk more than five blocks using minimal support.

\section{Conclusion}

We conclude that conversion of symptomatic hemiarthroplasty to THA is a safe option that gives good functional results, with marginally higher rates of intraoperative complications; and patients should be warned of the possibility of incomplete relief of groin postoperatively.

\section{References}

1. Yan WP, Chiu KY, Critical radiological analysis after Austin Moore hemiarthroplasty. Injury 2004, pn 10204.

2. Wachtl SW, Jacob RP, Gautier E, Ten-year patient and prosthesis survival after unipolar hip hemiarthroplasty in female patients over 70 years old. J Arthoplasty 2003; 18:587-91.

3. Clayer M, Brucknr J, The outcome of Austin-Moore hemiarthroplasty for fracture of the femoral neck. And orthop 1997: 26:681-4.

4. Pollegrini VD Jr, Heiges BA, Bixler B, Lehman EB, Davis $\mathrm{CM} 3^{\text {rd }}$. Minimum ten-year results of primary bipolar arthroplasty for degenerative arthritis of the hip.j Bone Joint Surg Am 2006;88:1817-25.

5. Raja PJ, Champan CB, Herrera MF, Schweppe MW, Michelsen CB, Rosenwasser MP. Unipolar or bipolar hemiarthroplasty for femoral neck fractures in the elderly? Clin othrop Relat Res 2003;414:259-69

6. Squires B, Bannister G. Displaced intracapsular neck femur fracture in mobile independent patients total hip replacement or hemiarthroplasty. Injury 1999; 30:345-8.

7. Hunter GA. Should we abandon primary prosthetic replacement for fresh displaced fractures of the neck of the lemur? Clin orthop Relat Res 1980; 152:158-61.

8. Sierra RJ, Cabanela ME. Conversion of failed hip hemairthroplasties after femoral neck fractures. Clin orthop Relat Res 2002; 399:129-39.

9. Hammad A, Abdel- Aal A. Conversion total hip airthroplasty: Functional outcome in Egyptain population. Acta Orthop Belg 2006; 72:549-54.

10. Bilgen $\mathrm{O}$, Karaeminogullari $\mathrm{O}$, Kulecioglu A. Results of conversion total hip prosthesis performed following painfull hemiarthroplasty. J lnt Med Res 2000; 28:30712. 